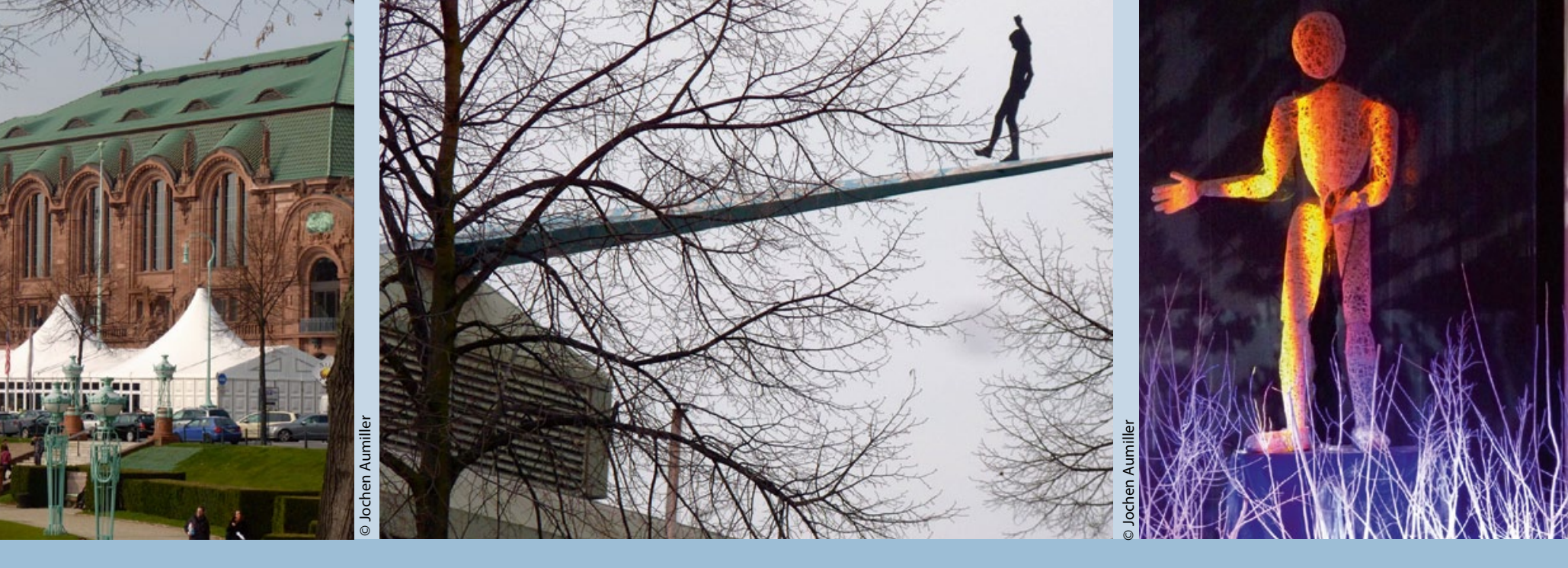

\title{
Rekordbeteiligung und Stafettenwechsel
}

Wieder konnte die DGK Rekorde melden: Knapp 8500 aktive Teilnehmer aus fast 30 Ländern haben die 79. Jahrestagung besucht. Erneut zeigte sich, dass das Mannheimer Kongresszentrum seine Kapazitätsgrenzen längst erreicht hat. Ein Wechsel des traditionellen Tagungsorts steht aber nicht zur Diskussion. In einigen Sitzungen reichten selbst Steh- und Sitzplätze auf dem Boden nicht aus. Auf internationalen Kongressen hat man dann Ausweichquartiere mit Videoübertragungen in petto, das scheint hier nicht machbar zu sein. 3729 Vorträge und Poster-Präsentationen waren im Angebot, als thematischen Schwerpunkt wählte Kongresspräsident Prof. Hugo Katus, Hei- delberg, die Herzinsuffizienz - die wohl größte Herausforderung der Kardiologie, in medizinischer und ökonomischer Hinsicht. Katus hat damit ein unübersehbares Signal gehisst. Er hätte natürlich auch seine wissenschaftliche Pionierleistung in den Mittelpunkt stellen können: das Troponin. Ein besonderes Zeichen setzte der scheidende DGK-Präsident Prof. Georg Ertl, Würzburg. Eine seiner letzten Amtshandlungen war die Gründung der Projektgruppe „Familie in der Kardiologie", womit die DGK „die Förderung des wissenschaftlichen Nachwuchses nachhaltig sichern möchte". Eine höchst brisante Initiative, denn der Nachwuchsmangel wird immer bedrückender. Viele gut ausgebil- dete Ärztinnen, so Ertl vor der Presse, steigen aus, wenn sie Mitte 30 sind und Kinder bekommen. Auch unter den männlichen Kollegen sei der Erziehungsurlaub keine Ausnahme mehr. Deshalb gilt es Teilzeitmodelle zu integrieren, wahrlich keine leichte Aufgabe.

Zum Ende der Tagung löste Prof. Christian W. Hamm Prof. Georg Ertl als DGKPräsident ab. Hamm ist ärztlicher Direktor und Geschäftsführer der Kerckhoff-Klinik in Bad Nauheim und Direktor der Medizinischen Klinik 1 des Universitätsklinikums Gießen. In der Mitgliederversammlung wurde Prof. Dr. Karl-Heinz Kuck, Hamburg, als Nachfolger von Hamm in zwei Jahren gewählt.

(J.A.) I

\section{Diagnostische Herausforderungen}

\section{Schwerpunktthema: Kardiomyopathie}

Tagungspräsident Prof. Dr. Hugo Katus, Ärztlicher Direktor der kardiologischen Abteilung am Universitätsklinikum Heidelberg, rückt die Kardiomyopathien in den Vordergrund, und zwar aus gutem Grund. Denn „bei der Hälfte aller Patienten mit einer Herzinsuffizienz liegt eine Kardiomyopathie vor".

6,5 Millionen Patienten in Europa leiden Katus zufolge an einer Herzinsuffizienz (HI). Pro Jahr kommen in Europa 600000 neue Fälle hinzu. Katus: „Überspitzt könnte man sagen: Dank der Fortschritte der modernen Herzmedizin überleben die Patienten zum Beispiel den Herzinfarkt und entwickeln in der Folge eine HI. Sie beeinträchtigt die Lebensqualität schwer - bis hin zur Invalidität
- und erzeugt erhebliche Kosten, in den USA waren es 201024 Milliarden USD." Die Prognosen sind trotz aller pharmakologischen und technischen Fortschritte nicht gut: 50\% der Patienten mit der Diagnose symptomatische $\mathrm{HI}$ sterben innerhalb von 4 Jahren, über 50\% der Menschen mit "schwerer" $\mathrm{HI}$ innerhalb eines Jahres. Bei HI kommt es 6- bis 9-mal so häufig zu einem tödlichen Herzstill- stand wie bei gesunden Menschen. Katus verwies auf "enorme Fortschritte in der Aufklärung der Ursachen der HI und Herzmuskelerkrankungen". Er unterschied dilatative, hypertrophische, restriktive und elektrische Kardiomyopathien und die Myokarditis.

Mit großem Aufwand werden derzeit die genetischen Faktoren aufgeklärt. Denn längst ist klar, dass die Kardiomyopathien in vielen Fällen erblich sind. Große Hoffnungen setzt man in die neuen molekularen Diagnoseverfahren; mit ihnen soll zukünftig besser zwischen verschiedenen Ursachen und Formen der HI differenziert werden können ein Schritt in Richtung individualisierte The- 